Martin Heger

Humboldt-Universität zu Berlin (Humboldt University of Berlin, Germany) e-mail: martin.heger@rewi.hu-berlin.de

ORCID: 0000-0001-5319-218X

DOI: 10.15290/mhi.2020.19.02.08

\title{
BGHSt 20, 22 und die Neubürger-Klausel des deutschen Strafanwendungsrechts - ein deutsch-polnischer Fall schreibt Rechtsgeschichte bis heute
}

\begin{abstract}
The judgment of the Bundesgerichtshof (Federal Court of Justice) from the 4th September 1964 and the "New Citizenship Clause" as part of the German law of jurisdiction in criminal cases - a German-Polish case and its implications for the legal development till today

In its judgment of the 4th September 1964 the German Federal Court of Justice had to deal with a German-Polish case of murder. The accused persons were members of the German minority in Poland and lived in the Western part of Poland, when German troops occupied that territory in the autumn of 1939. Short after the invasion they killed the members of a Jewish family living in the same territory. Both, the perpetrators as well as the victims were Polish nationals, when the crime was committed. The perpetrators have got the German nationality in the following. It is not clear, whether they have been naturalized by German authorities during WW II, but if not, they were seen as German nationals with the Getting-into-Force of the German Basic Law (Constitution), because they were refugees from Poland to Germany. Therefore, Article $116 \$ 1$ Basic Law naturalized them as German nationals. For the criminal case it was crucial whether the later naturalization can give the German Justice system jurisdiction over a case which happened before the perpetrator has reached the German nationality, as it was (and still is) stated in
\end{abstract}


section 4 (resp. today section 7) of the German Criminal Code. The paper should deal with the implications of this so-called "New Citizenship Clause“ and with the circumstances of the case and the following cases at Western German Courts against German people for committing murder in the occupied Polish territories during WW II. Shortly after the named case, the Auschwitz trial started in Frankfurt. On that background, the paper reflects on the situation between West and East Germany as well.

Key words: Federal Court of Justice, New Citizenship Clause, criminal cases, German-Polish case

\section{Zum Thema}

Das Strafanwendungsrecht - in traditioneller deutscher Diktion in terminologischer Anlehnung an das Internationale Privatrecht (IPR) das ,internationale Strafrecht" - der $\$ \$ 3 \mathrm{ff}$. StGB bestimmt seit jeher und bis heute darüber, auf welche Straftaten deutsches Strafrecht zur Anwendung kommen kann und damit zugleich, über welche Taten die deutsche Strafjustiz zu richten befugt ist. Indem mithin in materielle Hinsicht über die Strafbarkeit von (Auslands-)taten und in prozessualer Hinsicht über die Prozessvoraussetzung der Zuständigkeit der deutschen Justiz befunden wird, kommt den $\$ \$ 3 \mathrm{ff}$. StGB eine materiellrechtlich-prozessuale Doppelnatur zu. ${ }^{1}$

Schon im Lichte des Völkerrechts hängt die Anwendbarkeit deutschen Strafrechts zunächst von einer diesbezüglichen Normierung des deutschen Gesetzgebers, dann aber auch von einem sog. „genuin link“ des abzuurteilenden Falles zu Deutschland ab. So wäre eine universelle Erstreckung des deutschen Strafrechts auf alle Taten in der ganzen Welt ohne irgendeinen völkerrechtlich legitimierten Anknüpfungspunkt wegen des damit verbundenen Übergriffs in die nationale Souveränität des Tatortstaates evident völkerrechtswidrig. Dem Rechnung tragend beschränkt das deutsche Strafanwendungsrecht die Verfolgung von Auslandstaten grundsätzlich auf anerkannte Anknüpfungspunkte wie das Flaggen-, Schutz- und Personalitätsprinzip; nur in Ausnahmefällen ist deutsches Strafrecht universell anwendbar, wobei die Rechtsprechung zu $₫ 6$ StGB für die darin genannten Auslandstaten gegen international geschützte Rechtsgüter im Wege

1 Vgl. nur M. Heger, in: StGB, Kommentar, K. Lackner, K. Kühl, 29. Aufl. 2018, Vor $\$ \$ 3-7$ Rn. 10. - Zum Strafanwendungsrecht im deutschen Strafrechtssystem grundlegend F. Jeßberger, Der transnationale Geltungsbereich des deutschen Strafrechts, 2011, S. 108 ff.; aus rechtsvergleichender Sicht analysieren $\$ \$ 3 \mathrm{ff}$. StGB, M. Wörner, in: Jurisdiktionskonflikte bei grenzüberschreitender Kriminalität, A. Sinn (Hrsg.), 2012, S. 203 ff. 
völkerrechtskonformer Reduktion immer noch zumindest irgendeinen Bezugspunkt zu Deutschland fordert, ${ }^{2}$ auf den nur bei der Verfolgung von Völkerverbrechen nach dem deutschen Völkerstrafgesetzbuch (VStGB) nach $₫ 1$ Satz 1 VStGB verzichtet werden kann; im Unterschied zu $₫ 6$ StGB, der zwar keine explizite Beschränkung auf einen Bezug des Falles zu Deutschland enthält, einen solchen aber umgekehrt auch nicht explizit für irrelevant erklärt und damit für die erwähnte einschränkende Interpretation durch die Rechtsprechung offen ist, heißt es in $₫ 1$ Satz 1 VStGB ausdrücklich, das Völkerstrafgesetzbuch gelte für die darin enthaltenen Völkerverbrechen „auch dann, wenn die Tat im Ausland begangen wurde und keinen Bezug zum Inland aufweist". Da es sich bei diesen Delikten aber durchweg auch um im Statut von Rom für die Völkergemeinschaft als Völkerverbrechen ausgewiesene Straftaten handelt, an deren weltweiter Verfolgung notfalls auch gegen den Willen des Tatortstaates mithin ein genuines Interesse der Staatengemeinschaft besteht, ist der damit verbundene Eingriff in die Souveränität der Tatortstaaten auch ohne irgendeinen Bezug zu Deutschland von Völkerrechtswegen nicht zu beanstanden.

Der nachfolgend näher darzustellende Fall führt aber viel weiter zurück in die deutsch-polnische Geschichte, und zwar als schließlich angeklagter Lebenssachverhalt konkret in die ersten Monate nach dem deutschen Überfall auf Polen am 1. September 1939; der genaue Zeitpunkt der schließlich Anfang der 1960er Jahre am Schwurgericht in Stuttgart angeklagten Mordtaten lässt sich nicht exakt bestimmen, so dass letztlich offen bleiben muss, ob der von Deutschland dekretierte Anschluss des Gebietes um Posen zum 1.11.1939, des Tatorts des schließlich angeklagten Geschehens, auch im Sinne des bundesdeutschen Strafrechts eine Inlandstat bedeutet hätte. Vielmehr gehen das Schwurgericht Stuttgart und der Bundesgerichtshof (BGH) als oberste Instanz zugunsten der Angeklagten davon aus, dass der Tatort zur Tatzeit nicht zum Inland gehört hat und weder Opfer noch Täter damals (bereits) deutsche Staatsangehörige waren, so dass weder das Tatortprinzip noch das aktive oder passive Personalitätsprinzip im Grundsatz einen Anknüpfungspunkt für die Anwendbarkeit deutschen Strafrechts abgegeben haben. Es handelte sich - zumindest unter Anwendung von in dubio pro reo - mithin formal betrachtet (trotz der zu diesem Zeitpunkt längst bestehenden faktischen Kontrolle und Herrschaft deutscher Militär- und Zivilorgane in diesem Teil Polens) um einen „klassischen“ Fall einer Straftat ohne Inlandsbezug: Ein Ausländer ermordet Ausländer im Ausland. Normalerweise fehlt es in einer solchen Konstellation gerade an irgendeinem „genuin link“ zu Deutschland. Umgekehrt wäre natürlich eine Aburteilung im Tatortstaat - hier also in Polen - denkbar gewesen.

$2 \quad$ BGHSt 27, 30; 34, 334; Heger (Fn. 1), $₫ 6$ Rn. 1. - Tendenziell weiter für $₫ 6$ Nr. 9 StGB etwa BGHSt 45, 64 und 46, 292. 
Dies war hier freilich praktisch unmöglich, denn die Täter waren längst in der Bundesrepublik Deutschland. Vorliegend wäre es nicht bloß ein kaum lösbares Politikum gewesen, die Täter an die - im Kommunismus nicht unabhängige - polnische Justiz auszuliefern; es war vor allem auch rechtlich zwischenzeitlich nach Inkrafttreten des Grundgesetzes am 23.5.1949 ein „Ding der Unmöglichkeit", denn Art. 16 Abs. 2 Grundgesetz (GG) statuierte damals - bis zur Einfügung einer Auslieferungsmöglichkeit an EU-Mitgliedstaaten aufgrund eines Europäischen Haftbefehls sowie an den Internationalen Strafgerichtshof im Jahr 2000 - ebenso knapp wir klar: „Kein Deutscher darf an das Ausland ausgeliefert werden“. Und wer Deutscher in diesem Sinne war und ist, bestimmt seit jeher Art. 116 Abs. 1 GG: „Deutscher im Sinne dieses Grundgesetzes ist vorbehaltlich anderweitiger gesetzlicher Regelung, wer die deutsche Staatsangehörigkeit besitzt oder als Flüchtling oder Vertriebener deutscher Volkszugehörigkeit oder als dessen Ehegatte oder Abkömmling in dem Gebiete des Deutschen Reiches nach dem Stande vom 31. Dezember 1937 Aufnahme gefunden hat.“

Das Schwurgericht konnte nicht sicher feststellen, ob die beiden Angeklagten L. und R. tatsächlich bis zum Prozess die deutsche Staatsangehörigkeit erlangt hatten. Darauf kam es aber auch nicht entscheidend an, weil sie nach dem zweiten Weltkrieg als Flüchtlinge offenbar in Westdeutschland und damit im vormaligen, in Art. 116 Abs. 1 GG genannten Reichsgebiet Aufnahme gefunden hatten. Da sie zuvor - außerhalb der deutschen Grenzen von 1937 - in Polen als Angehörige der deutschen Minderheit gelebt hatten, waren sie zumindest „deutsche Volkszugehörige“ im Sinne von Art. 116 Abs. 1 GG und damit spätestens mit dessen Inkrafttreten 1949 auch Deutsche. Vor diesem Hintergrund ging es im nachfolgend näher darzustellenden Fall vor allem darum, ob und mit welchen Folgen die heute in $\$ 7$ Abs. 2 Nr. 1 StGB (bis $1975 \$ 4$ Abs. 2 Nr. 1 StGB) enthaltene sog. „Neubürger-Klausel“ als Extension des aktiven Personalitätsprinzips die Anwendbarkeit deutschen Strafrechts und damit die Zuständigkeit der deutschen Strafjustiz für den vorliegenden Fall begründen kann.

Neben der Tatzeit ist aber auch die Zeit des Strafverfahrens nicht ohne Relevanz, geht es doch um ein Anfang der 1960er Jahre und damit in der Hochzeit des "kalten Krieges“ von der bundesdeutschen Justiz betriebenes Strafverfahren. In dieser Zeit kurz nach dem Bau der Berliner Mauer und einige Jahre vor dem Start der "neuen Ostpolitik“, die in Bezug auf das Polen in der Unterzeichnung des Warschauer Vertrages am 7.12.1970 gipfeln sollte, herrschte zwischen der noch stark antikommunistisch ausgerichteten westdeutschen Regierung und den kommunistischen Regimes im Ostblock, zu denen auch Polen zählte, politisch weitgehend Eiszeit; angesichts der die Ostpolitik seit 1955 beherrschenden Hallstein-Doktrin bestanden zwischen beiden Staaten nicht einmal diplomatische Beziehungen. Allerdings gab es im Spätjahr 1963, als in Stuttgart der Strafprozess in erster Instanz durchgeführt wurde, bereits erste 
Aufweichungen dieser Doktrin in Folge des Rücktritts von Konrad Adenauer als Bundeskanzler und der Übernahme dieses Amtes durch Ludwig Erhard am 17.10. 1963.

\section{Zum abzuurteilenden Fall}

Der der Aburteilung vor dem Schwurgericht am Landgericht Stuttgart zugrunde liegende Fall lässt sich grob aus einzelnen tatsächlichen Angaben rekonstruieren, die - soweit für die rechtliche Bewertung in der Revision erforderlich - in die Rechtsausführungen des 1. Strafsenats des BGH eingeflochten sind und die ich vorab kurz zusammenfassen möchte. Die beiden Angeklagten L. und R., die gegen ihre Verurteilung vor dem BGH als Revisionsführer agierten, waren offenbar Ende der 1930er Jahre in Polen lebende Angehörige der deutschen Minderheit, die kurz nach der Okkupation Polens durch deutsche Truppen vier Mitglieder einer jüdischen Familie - wie die Täter offenbar bis dahin polnische Staatsangehörige - ermordet haben. Noch während des zweiten Weltkrieges oder in den Jahren danach sind sie dann offenbar als Flüchtlinge nach Westdeutschland gekommen (interessant ist, dass im vorliegenden Urteil nicht der damals für Flüchtlinge aus früher deutschen Gebieten gängige Terminus der „Heimatvertriebenen“ oder - kurz - „Vertriebenen“ gebraucht worden ist). Der Terminus „Flüchtlinge“ macht allerdings deutlich, dass die beiden Angeklagten nicht etwa bereits vor Kriegsende aus freien Stücken aus Posen nach (West-) Deutschland umgesiedelt sind, sondern entweder vor der vorrückenden Roten Armee geflohen oder - wie in der unmittelbaren Nachkriegszeit üblich - als „Volksdeutsche" von den polnischen Behörden zur Ausreise gezwungen worden sind oder nach der Wiedererrichtung des polnischen Staates angesichts der bereits 1944 in einem polnischen Dekret für solche Taten angedrohten Todesstrafe das Land verlassen haben.

Inhaltlich geht es um frühe und vergleichsweise „wilde“, d.h. noch nicht massenhaft gleichsam industriell betriebene Judenvernichtung bereits kurz nach dem Einmarsch der deutschen Truppen, aber - im Unterschied zu den heute vielfach auf Fotos erkennbaren Gewalt- und Diskriminierungsakten von Angehörigen der deutschen Wehrmacht oder der dieser folgenden Polizeibataillone gegenüber osteuropäischen Juden - eben auch nicht um eine von oben angeordnete oder strukturierte Maßnahme, sondern um den Ausbruch massiver Gewalt von deutschstämmigen Bürgern Polens gegenüber ihren langjährigen jüdischen Mitbürgern angesichts der veränderten politischen Rahmenbedingungen. Anders als bei den Nürnberger Kriegsverbrecher-Prozessen der Alliierten, aber auch dem Ulmer Einsatzgruppen-Prozess von 1957 und den Mitte der 1960er Jahre in Frankfurt stattfindenden Auschwitz-Prozessen sowie den Verfahren vor dem Oberste Nationale Tribunal Polens (Najwyższy Trybunał Narodowy, NTN) u.a. gegen KZ-Kommandanten und andere hohe Vertreter der 
deutschen Besatzungsmacht in Polen (1946-1948) und schließlich - kurz vor dem hier zu besprechenden Fall - dem Prozess gegen Eichmann in Jerusalem ging es damit vorliegend nicht um staatlich organisierten oder zumindest veranlassten Massenmord, sondern um individuelle Mordtaten, die aber ideologisch der NS-Doktrin entsprachen und aufgrund der politischen Begünstigung durch die deutsche Besatzungsmacht zur Tatzeit und in den Jahren unmittelbar danach sanktionslos bleiben mussten.

Bemerkenswert ist, dass die Justiz bis zur Verkündung des erstinstanzlichen Urteils offenbar keine Haftgründe erblickt hat und deswegen die beiden Angeklagten trotz der gegen sie verhandelten Mordanklage bis zum 20.12.1963, dem Tag der Urteilsverkündung, anscheindend auf freiem Fuß geblieben sind; danach wurden sie in Untersuchungshaft genommen, wie sich daraus erhellt, dass in der Entscheidung des BGH nur von einer Anrechnung der Untersuchungshaft seit dem 21.12.1963 auf die verhängten Freiheitsstrafen von 15 Jahren (gegen L.) und 10 Jahren (gegen R.) die Rede ist. Aus heutiger Sicht muss das überraschen, denn in $\$ 112$ Abs. 3 StPO ist festgehalten, dass beim dringenden Tatverdacht bestimmter besonders schwerer Straftaten (u.a. Mord) eine Untersuchungshaft auch dann angeordnet werden kann, wenn kein zusätzlicher Haftgrund (Flucht, Fluchtgefahr oder Verdunkelungsgefahr) vorliegt. Diese Bestimmung ist aber erst kurz nach dem vorliegenden Prozess mit der sog. „kleinen Strafprozeßnovelle" vom 19.12.1964 ${ }^{3}$ mit Wirkung zum 1.4.1965 - vor allem zur Anwendung gegen NS-Verbrecher - in das Verfahrensrecht aufgenommen worden. ${ }^{4}$ Anscheinend hat das Schwurgericht angesichts der verhängten langjährigen Haftstrafen Fluchtgefahr angenommen; dass es zuvor nicht zu einer Inhaftierung gekommen sein dürfte, legt wohl nahe, dass bis zum Abschluss des Hauptverfahrens dessen Ausgang nicht so sicher geschienen haben mag, womit entweder bis zum Schuldspruch ein dringender Tatverdacht auszuscheiden hatte oder weit wahrscheinlicher - die Angeklagten aus formalen Gründen etwa mangels Anwendbarkeit des deutschen Strafrechts oder wegen des Nichtvorliegens eines Strafantrags der polnischen Behörden letztlich bis zum Schuldspruch darauf hoffen konnten, trotz ihnen nachzuweisender Tatbegehung ungeschoren davon zu kommen und deshalb keinen Anreiz zu einer Flucht hatten. Wäre Fluchtgefahr schon aus persönlichen Gründen (etwa wegen gesundheitlicher Probleme oder besonderer Ortsverbundenheit) auszuscheiden gewesen, hätte ja auch die zwischenzeitlich verhängte, aber noch nicht in Rechtskraft erwachsene Freiheitsstrafe keinen Fluchtgrund abgeben können.

3 Dazu P. Rieß, ZIS 2009, 466, 470.

$4 \$ 112$ Abs. 3 StPO hatte zunächst folgenden Wortlaut: „Gegen den Beschuldigten, der eines Verbrechens wider das Leben nach den $\$ \$ 211,212$ oder $\$ 220$ a Abs. 1 Nr. 1 des Strafgesetzbuches dringend verdächtig ist, darf die Untersuchungshaft auch angeordnet werden, wenn ein Haftgrund nach Absatz 2 und 3 nicht besteht.“ 
Von Interesse ist der Fall auch in zeitlicher Hinsicht, gelangte er doch Anfang der 1960er Jahre vor Gericht, zu einer Zeit also, als die Strafverfolgung von NS-Verbrechen zwar - nach dem Ulmer Einsatzgruppenprozess $1958^{5}$ und der Gründung der Zentralen Ermittlungsstelle für NS-Verbrechen 1958 in Ludwigsburg $^{6}$ - wieder angelaufen war, die großen Auschwitz-Prozesse ${ }^{7}$ aber erst vorbereitet wurden. Der Schuldspruch des Stuttgarter Schwurgerichts datiert vom 20.12.1963, just dem Tag, an dem in Frankfurt am Main der erste AuschwitzProzess begonnen hat. Obwohl - wie bei den Auschwitz-Prozessen - der Tatort zur Zeit der gerichtlichen Hauptverhandlung in Polen gelegen hat, gab es in dem vorliegenden Stuttgarter Strafprozess offenbar keinen Ortstermin; angesichts des erwähnten Fehlens diplomatischer Beziehungen aufgrund der Hallstein-Doktrin wäre ein solcher auch - wie sich dann im ersten Frankfurter Auschwitz-Prozess, wo es zu einem Ortstermin im früheren Vernichtungslager gekommen ist, herausstellen sollte - organisatorisch nur schwer zu bewerkstelligen gewesen.

\section{Zur Entscheidung}

Das vom 1. Strafsenat am 8.9.1964 unter dem Aktenzeichen 1 StR 292/64 verkündete Urteil in der Revision, das - und das spricht natürlich für seine schon aus zeitgenössischer Sicht bleibende Bedeutung - unter der Kurzzitierung BGHSt 20, 22 Eingang in die amtliche Sammlung gefunden hat, hat (gekürzt) folgenden Wortlaut:

Das Schwurgericht hat die Angeklagten - unter Freisprechung und Einstellung des Verfahrens im übrigen - wegen gemeinschaftlichen vierfachen Mordes zu zeitigen Zuchthausstrafen verurteilt und ihnen die bürgerlichen Ehrenrechte auf eine ihrer Strafe entsprechende Zeit aberkannt. Dagegen haben die Angeklagten - sinngemäß, soweit sie verurteilt worden sind - Revision eingelegt. Die Rechtsmittel führen zu einer Berichtigung des Urteils, haben in Ergebnis aber keinen Erfolg.

1. Auf die Tat ist zunächst deutsches Strafrecht anzuwenden.

Das bestimmt $₫ 4$ Abs. 2 Nr. $1 \mathrm{StGB}^{8}$ in der jetzt geltenden Fassung. Die Vorschrift ordnet die Anwendung deutschen Strafrechts auf eine von einem

5 Dazu: S. Müller, T. John, Die Mörder sind unter uns: Der Ulmer Einsatzgruppenprozess 1958, 2008.

6 Zu deren Geschichte vgl. den Bericht ihrer damaligen Leiter Schramm, Riedel, „Vierteljahreshefte für Zeitschichte" 2008 (VfZ), 4, 525 ff.

7 Dazu ausführlich G. Werle, T. Wandres, Auschwitz vor Gericht, 1995.

8 Von 1957 bis 1968 und damit zur Zeit des Strafverfahrens hatte $₫ 4$ Abs. 2 Nr. 1 StGB folgenden Wortlaut: „Für eine von einem Ausländer im Ausland begangene Straftat gilt das deutsche Strafrecht, wenn sie durch das Recht des Tatorts mit Strafe bedroht oder der Tatort keiner Strafgewalt unterworfen ist und wenn 1. der Täter die deutsche Staatsangehörigkeit nach der Tat erworben hat". Dies entspricht in der Sache dem heutigen $₫ 7$ Abs. 2 Nr. 1 StGB, der wie folgt lautet: „Für andere Taten, die im Ausland begangen werden, gilt das deutsche Strafrecht, wenn die Tat am Tatort mit Strafe bedroht ist oder der Tatort keiner Strafgewalt unterliegt und wenn der Täter 1. zur Zeit der Tat Deutscher war oder es nach der Tat geworden ist“. 
Ausländer im Ausland begangene und nach dem Recht des Tatorts mit Strafe bedrohte Handlung an, wenn der Täter nach der Tat die deutsche Staatsangehörigkeit erworben hat. Sie will so verhindern, dass der Rechtsbruch des Täters, der als Deutscher nach Art. 16 Abs. 2 Satz 1 GG nicht ausgeliefert werden darf, ungesühnt bleibt., Verfassungsrechtlichen Bedenken begegnet diese Regelung nicht, $\$ 4$ Abs. 2 Nr. 1 StGB bestimmt zwar wegen eines erst nach der Tat eingetretenen Umstandes, nämlich wegen des Erwerbs der deutschen Staatsangehörigkeit, dass auf das Geschehen deutsches Strafrecht anzuwenden ist. Er verstößt deshalb aber nicht gegen Art. 103 Abs. 2 GG, nach dem zur Tatzeit bestimmt sein muss, ob eine Handlung strafbar ist und mit welcher Strafe sie bedroht ist; denn zur Tatzeit stand fest, dass und wie die Tat zu bestrafen ist, wenn der Täter nach ihrer Begehung deutscher Staatsangehöriger wird (Stree, Deliktsfolgen und Grundgesetz S. 32 f).

Das Schwurgericht hat das Verhalten der Angeklagten im Ergebnis mit Recht als eine von Ausländern gegenüber Ausländern im Ausland begangene Tat behandelt. Nach den Feststellungen über Abstammung, Aufenthalt und Wehrpflicht waren sowohl die Opfer als auch mindestens bis zum 26. Oktober 1939 die Beschwerdeführer polnische Staatsangehörige. Der Tatort gehörte mindestens bis zum 31. Oktober 1939 zu Polen. Ob die Angeklagten auf Grund der während der deutschen Besetzung Polens erlassenen, staatsrechtlich wirksamen ${ }^{9}$ Vorschriften die deutsche Staatsangehörigkeit erworben haben und ob dies strafrechtlich rückwirkend vom 26. Oktober 1939 an Bedeutung hätte, kann unerörtert bleiben. Ebenso braucht nicht dazu Stellung genommen zu werden, ob der Tatort durch die Erklärung Posens zum deutschen Reichsgebiet (Erlass vom 8. Oktober 1939 - RGBl. I S. 2042) vom 1. November 1939 an vorübergehend strafrechtlich Inland geworden ist. Die Angeklagten haben die strafbare Handlung Ende Oktober/Anfang November 1939, bestimmt vor dem 15. November 1939 begangen. Genauer hat sich die Tatzeit nicht mehr ermitteln lassen. Daher kann nicht ausgeschlossen werden, dass sich die Tat schon kurz vor dem 26. Oktober 1939 zugetragen hat. Von dieser Möglichkeit muss ausgegangen werden. Sie ist für die Beschwerdeführer die günstigste; denn dann haben sie als Ausländer gegenüber Ausländern im Ausland gehandelt und ihnen kommen mildere polnische Bestimmungen zugute, deren Anwendung in diesem Fall vorgeschrieben ist.

Ob die Angeklagten jetzt deutsche Staatsangehörige sind, hat das Schwurgericht nicht zuverlässig klären können. Das schadet nicht. Der Tatrichter hat

9 Vgl. BVerfGE 1, 322, 328 f.; 2, 115; 14, 142; ferner $\$ 1$ Abs. 1 Buchst. d des Gesetzes zur Regelung von Fragen der Staatsangehörigkeit vom 22. Februar 1955 - BGBl I S. 65 - und das polnische Dekret vom 13. September 1946, betreffend den Ausschluss von Personen deutscher Nationalität aus der polnischen Volksgemeinschaft, in deutscher Übersetzung abgedruckt in Geilke, Das Staatsangehörigkeitsrecht von Polen, S. 106. 
zutreffend beide Beschwerdeführer, Flüchtlinge „deutscher Volkszugehörigkeit“, als Deutsche im Sinne des Art. 116 Abs. 1 GG angesehen und sie deshalb bei der Entscheidung, ob ihr Verhalten nach deutschem Strafrecht zu ahnden ist, mit Recht ebenso wie solche Täter behandelt, die die deutsche Staatsangehörigkeit nach der Tat erworben haben (BGHSt 11, 63).

Das Recht des Tatorts bedroht die Tat mit Strafe; denn wer Menschen vorsätzlich tötet, wird nach dem zur Tatzeit und auch jetzt noch geltenden (vgl. ROW 1963, 70) Art. 225 poln. StGB mit dem Tode oder mit Freiheitsentzug bestraft.

2. Dem Verfahren stehen keine Hindernisse entgegen.

a) Entgegen der Meinung der Revision ist ein Antrag der zuständigen polnischen Behörde auf Verfolgung der Beschwerdeführer nicht erforderlich.

Das nach $₫ 4$ Abs. 2 Nr. 1 StGB anzuwendende deutsche Recht schreibt vor, dass sich die Strafe grundsätzlich nach dem Gesetz bestimmt, das zur Zeit der Tat gilt. Bei Verschiedenheit der Gesetze von der Zeit der begangenen Handlung bis zu deren Aburteilung ist das mildeste Gesetz anzuwenden ( $\$ 2$ Abs. 2 StGB). $\mathrm{Zu}$ den Gesetzen in diesem Sinn gehört auch $₫ 4$ Abs. 2 StGB. Die Regelung, ob auf ein bestimmtes Geschehen das deutsche Strafrecht anzuwenden ist, ist nämlich - von wenigen Einzelheiten abgesehen - ein Teil des sachlichen Rechts; sie behandelt das Ausmaß der Geltung des sachlichen Strafrechts und befasst sich nicht mit der verfahrensrechtlichen Frage nach dem Umfang der Strafgerichtsbarkeit (Schäfer in Löwe/Rosenberg, StPO 21. Aufl. Einl. S. 82; Jescheck, Internationales Recht und Diplomatie 1956 S 76; Hegler, Prinzipien des internationalen Strafrechts S. 17 ff). Das mildeste Gesetz ist durch einen Vergleich der verschiedenen Rechtszustände von der Zeit der Tat bis zur Zeit der Aburteilung zu ermitteln. Dabei sind nicht nur die Tatbestände und die Strafdrohungen der verschiedenen Gesetze abstrakt aneinander zu messen; maßgebend ist vielmehr, welche Regelung in dem zu entscheidenden Einzelfall nach dessen besonderen Umständen die dem Täter günstigere Beurteilung zulässt (RGSt 61, 76 f und 130, 135; 71, 42 f; 75, 306, 310; BGH LM StGB $\$ 200$ Nr. 2).

$\$ 4$ Abs. 2 StGB galt zur Tatzeit in der Fassung vom 24. April 1934. Nach Nr. 3 der Vorschrift konnte auf eine Auslandstat eines Neubürgers, die nach deutschem Recht als Verbrechen oder Vergehen anzusehen und durch die Gesetze des Tatorts mit Strafe bedroht war, deutsches Strafrecht angewendet werden. Die Verfolgung bedurfte jedoch eines Antrags der zuständigen Behörde des Landes, in dem die Tat begangen worden war, und das ausländische Strafgesetz war anzuwenden, soweit es milder war. Nach der durch die Verordnung über den Geltungsbereich des Strafrechts vom 6. Mai 1940 (GeltungsbereichsVO) eingeführten und jetzt geltenden Fassung des $₫ 4$ Abs. 2 Nr. 1 StGB ist jede Auslandstat eines Neubürgers nach deutschem Strafrecht zu ahnden, wenn die 
Handlung durch das Recht des Tatorts mit Strafe bedroht ist. Milderes ausländisches Recht ist nicht mehr anzuwenden, und ein Antrag der zuständigen Behörde des Landes, in dem die strafbare Handlung begangen wurde, ist nicht mehr erforderlich. Die neue Regelung ist im vorliegenden Fall strenger. Nach ihr wäre das Verhalten der Beschwerdeführer nach deutschem Recht zu beurteilen und als gemeinschaftlicher Mord sowohl im Sinne des $\$ 211$ StGB aF als auch im Sinne des $₫ 211$ StGB nF nach $\$ 211$ StGB nF mit lebenslangem Zuchthaus zu bestrafen. Nach $₫ 4$ Abs. 2 Nr. 3 StGB in der zur Tatzeit geltenden Fassung kommt es zunächst auf einen Vergleich zwischen dem deutschen und dem polnischen Recht an. Das polnische Strafgesetzbuch kennt - von einigen hier nicht in Betracht kommenden begünstigten Fällen abgesehen - nur den Grundtatbestand der vorsätzlichen Tötung; im Gegensatz zum deutschen Strafrecht unterscheidet es nicht zwischen einer gewöhnlichen und einer besonders schwerwiegenden vorsätzlichen Tötung (Art. $225 \$ 1$ ). Die vorsätzliche Tötung bedroht es mit der Todesstrafe, lebenslangem oder zeitigem Freiheitsentzug. Das für Taten der vorliegenden Art die Todesstrafe vorschreibende polnische Dekret vom 31. August 1944 (in deutscher Übersetzung abgedruckt in Geilke, Die polnische Strafgesetzgebung seit 1944 S. 85) muss außer Betracht bleiben. Das schreibt den deutschen Gerichten schon das zum Verfassungssatz erhobene und auch bei der Anwendung ausländischen Rechts zu beachtende Verbot rückwirkender Strafgesetze (Art. 103 Abs. 2 GG) vor, abgesehen davon, dass nach polnischem Recht ebenfalls Strafbestimmungen in der Regel keine rückwirkende Kraft beigelegt werden darf (Art. 1, $2 \$ 1$ poln. StGB). Demnach ist hier im Vergleich zu dem für Mord zwingend lebenslanges Zuchthaus vorschreibenden deutschen Strafrecht das polnische Strafrecht für die Angeklagten günstiger, da nach ihm die Tat auch durch eine zeitige Freiheitsstrafe geahndet werden kann. Daraus ergibt sich zugleich, dass der die Anwendung dieses Rechts bestimmende $\$ 4$ Abs. 2 Nr. 3 StGB aF milder ist als $₫ 4$ Abs. 2 Nr. 1 StGB nF, nach dem die Beschwerdeführer nach deutschem Recht mit lebenslangem Zuchthaus zu bestrafen wären. Im Ergebnis ist das Schwurgericht daher mit Recht von $₫ 4$ Abs. 2 Nr. 3 StGB aF ausgegangen.

Nach dieser Vorschrift liegen alle Voraussetzungen für ein Einschreiten der deutschen Strafgerichte vor bis auf den nach ihr erforderlichen Antrag der Republik Polen auf Verfolgung der Tat. Dessen Fehlen hat der Tatrichter jedoch entgegen der Meinung der Revision mit Recht für entbehrlich gehalten. Das nach $\$ 4$ Abs. 2 Nr. 3 StGB aF notwendige Verlangen des ausländischen Staates nach Bestrafung des Täters ist zwar kein Strafantrag im Sinne des $₫ 61$ StGB; im Gegensatz zu den übrigen Merkmalen des $₫ 4$ Abs. 2 Nr. 3 StGB aF ist es aber ebenso wie jener (BGHSt 6, 155) eine Voraussetzung der Strafverfolgung, die in die sonst sachlichrechtliche Bestimmung eingefügt ist, um die nachträgliche Ahndung von Fällen auszuschließen, an der der Staat, dessen Rechtsordnung 
gebrochen worden ist, kein Interesse hat (RGSt 16, 216; vgl. \$ 104 a StGB). Der Antrag des ausländischen Staates ist daher kein sachlichrechtliches Erfordernis für die Anwendbarkeit deutschen Strafrechts, sondern eine Verfahrensvoraussetzung (von Bar, Gesetz und Schuld im Strafrecht Band 3 S. 364; Hugo Meyer, Lehrbuch des Deutschen Strafrechts 5. Aufl. S 293; Olshausen/Niethammer, StGB 11. Aufl. \$ 4 Anm. 18). (...)

Das Verbot rückwirkender Strafgesetze gilt nur für das sachliche Recht. Verfahrensvoraussetzungen hingegen treten mit dem Gesetz, das sie aufhebt, außer Kraft (RGSt 75, 306, 311). Da nach $₫ 4$ Abs. 2 Nr. 1 StGB nF die Befugnis deutscher Gerichte, gegen Auslandstaten von Neubürgern einzuschreiten, nicht mehr von einem Strafverlangen des Staates, in dem die strafbare Handlung begangen worden ist, abhängig macht, hat das Schwurgericht mit Recht einen Antrag der Republik Polen auf Verfolgung der Beschwerdeführer nicht für erforderlich erachtet. (...)

b) Die Strafverfolgung der Tat der Angeklagten ist weder nach deutschem noch nach polnischem Recht verjährt. Ihre Verfolgung fällt auch unter kein Straffreiheitsgesetz. Das hat das Schwurgericht richtig dargetan.

\section{3. (..)}

4. Die Sachrüge führt zu einer Berichtigung des Schuldspruchs und des Ausspruchs über den Ehrenrechtsverlust. Sonst hat sie ebenfalls keinen Erfolg.

a) Das Schwurgericht hat den Schuldspruch auf deutsches und den Strafausspruch in der Hauptsache auf polnisches Recht gegründet. Das ist nicht statthaft.

Zutreffend hat das Schwurgericht erwogen, dass das Verhalten beider Beschwerdeführer den Tatbestand des $\$ 211$ StGB sowohl in der zur Tatzeit geltenden als auch in der jetzt gültigen Fassung erfüllt. Es hat ohne Rechtsfehler auseinandergesetzt, dass keinem der Angeklagten ein Rechtfertigungs-, Schuldoder Strafausschließungsgrund zur Seite steht. Bei der eingehenden und sorgfältigen Prüfung, ob die Beschwerdeführer als Mittäter oder als Gehilfen gehandelt haben, hat es die für die Abgrenzung von Täter- oder Mittäterschaft und Gehilfenschaft entwickelten Grundsätze beachtet. Die dabei angestellten Überlegungen stehen auch nicht mit der Feststellung in Widerspruch, daß der Angeklagte L. es als eine Sache der nationalsozialistischen Machthaber ansah, dass sie Menschen allein wegen ihres Judentums töten ließen. Diese Feststellung besagt nämlich nur, dass L. sich über das Fehlen jedes, wenn auch noch so fadenscheinigen Beweggrundes für die unmenschliche Tat keinerlei Gedanken gemacht hat. Damit ist es durchaus vereinbar, dass er um persönlicher Vorteile willen den schweren Rechtsbruch, den er begehen sollte und dessen Ausführungsweise er zu bestimmen hatte, in seinen Willen aufgenommen und 
im Gefühl der persönlichen Verantwortung für das Gelingen in die Tat umgesetzt hat. (...)

Ohne Rechtsfehler zum Nachteil der Beschwerdeführer hat das Schwurgericht die Tat auch nach polnischem Recht als eine gemeinschaftliche vorsätzliche Tötung, Verbrechen nach Art. $225 \$ 1$ poln. StGB, gewertet. Das ergibt sich zweifelsfrei aus dem Zusammenhang seiner Ausführungen zur Schuld- und Straffrage. Der Tatrichter hat diesen Gesichtspunkt in dem Urteil freilich nicht besonders erörtert, weil er hinsichtlich der Schuldfrage die polnischen Bestimmungen nicht für milder gehalten und deshalb geglaubt hat, den Schuldspruch auf deutsches Recht stützen zu können, während er dem Strafausspruch in der Hauptsache polnisches Recht zugrunde gelegt hat. Diese Ansicht kann der Senat nicht billigen.

$\S 4$ Abs. 2 Nr. 3 StGB aF bestimmt, dass das ausländische Strafrecht anzuwenden ist, soweit es milder ist. Die Anordnung, das mildere fremde Recht anzuwenden, stellt klar, dass die deutschen Gerichte im Verurteilungsfall nicht etwa befugt sind, eine Strafe nach deutschem Recht zu verhängen, die nur das nach ausländischem Recht verwirkte Maß nicht übersteigen darf; sie müssen ihrer Entscheidung in solchem Fall vielmehr stets das ausländische Recht zugrunde legen, wenn dieses milder ist, und zwar im Ganzen. Um festzustellen, welches Recht milder ist, müssen die Gerichte den Sachverhalt jeweils vollständig sowohl nach den deutschen als auch nach den ausländischen Vorschriften untersuchen. Bei der Ermittlung des milderen Rechts i.S. des $₫ 4$ Abs. 2 Nr. 3 StGB aF kommt es nämlich ebenso wie bei $\$ 2$ Abs. 2 StGB nicht allein auf die allgemein angedrohten Strafen der verschiedenen Gesetze an; maßgebend ist vielmehr auch hier, welche Regelung in dem zu entscheidenden Einzelfall nach dessen besonderen Umständen die den Täter schonendere Beurteilung gestattet (Lobe in LK 5. Aufl. \$ 4 Anm. 3 Nr. 3 b; Olshausen/Niethammer, StGB 11. Aufl. $\$ 4$ Anm. 19). Das kann man nur feststellen, indem man den Sachverhalt jeweils getrennt vollständig den einschlägigen Strafgesetzen der beiden zu vergleichenden Rechtsordnungen unterordnet. Die Regelung, die sich bei diesem Vergleich als für den Täter günstiger erweist, muss dann im ganzen angewendet werden. Das hat das Reichsgericht in den Fällen, in denen zur Tat- und zur Aburteilungszeit verschiedene deutsche Gesetze galten, stets angenommen (RGSt 58, 238; 61, 76; 74, 132, 133; 77, 219; RG DR 1944, 367). Dasselbe muss gelten, wenn bei einer Auslandstat eines Neubürgers das deutsche und das Recht des Tatorts voneinander abweichen und nach $\$ 4$ Abs. 2 Nr. 3 StGB aF das mildere ausländische Recht anzuwenden ist. Auch in diesem Fall ist es nicht vertretbar, die in dem einen Strafgesetz angeordneten Folgen losgelöst von den in ihm bestimmten Voraussetzungen aufgrund der Bedingungen der anderen aus einer fremden Rechtsordnung stammenden Vorschrift auszusprechen.

Das Versehen des Tatrichters kann jedoch behoben worden. Die Ausführungen zum Strafausspruch lassen deutlich erkennen, dass das Schwurgericht das 
Verhalten der Beschwerdeführer auch nach polnischem Recht untersucht hat. Es hat darin ohne Rechtsirrtum zum Nachteil der Angeklagten eine gemeinschaftliche vorsätzliche Tötung, Verbrechen nach Art. $225 \$ 1$ poln. StGB, gesehen und zu deren Ahndung wegen der bisherigen Unbescholtenheit der Angeklagten und wegen der ganz außergewöhnlichen Tatumstände mit Recht zeitige Freiheitsstrafen für angemessen erachtet. Dieses im Vergleich zu $\$ 211$ StGB nF, nach dem die Beschwerdeführer lebenslanges Zuchthaus verwirkt hätten, mildere polnische Recht ist nach $\$ 4$ Abs. 2 Nr. 3 StGB aF allein anzuwenden und sowohl dem Schuld- als auch dem Strafausspruch zugrundezulegen.

b) Mit Recht hat das Schwurgericht auf Zuchthaus- und nicht auf Gefängnisstrafen erkannt. Die deutsche Übersetzung bezeichnet die in Art. $225 \$ 1$ poln. StGB angedrohte Freiheitsstrafe zwar mit „Gefängnis“. Das besagt jedoch nicht, dass damit auch Gefängnis im Sinne des deutschen Freiheitsstrafensystems gemeint ist. Das polnische Strafgesetzbuch kennt nur zwei Arten von Freiheitsstrafen, die in den deutschen Übersetzungen "Gefängnis" und „Haft" genannt werden (Art. 37 poln. StGB). Die "Gefängnisstrafe“ dauert mindestens sechs Monate und längstens 15 Jahre, wenn das Gesetz nicht im Einzelfall lebenslange Dauer vorsieht. Sie wird nach Monaten und Jahren bemessen. Der zu ihr Verurteilte muss die ihm zugewiesenen Arbeiten verrichten, auch außerhalb der Anstalt (Art. 39, 41 poln. StGB). „Haft“ kann von einer Woche bis zu fünf Jahren verhängt werden. Die Strafe wird nach Wochen, Monaten und Jahren bemessen. Wer zu „Haft" verurteilt worden ist, braucht von der Anstaltsleitung zugewiesene Arbeiten nur auszuführen, wenn er sich während dar Strafverbüßung nicht nach eigener Wahl selbst beschäftigt (Art. 40, 41 poln. StGB). „Gefängnis“ von mehr als fünf Jahre ist Verbrechens- und "Gefängnis" bis zu fünf Jahren und „Haft“ sind Vergehensstrafen (Art. 12 poln. StGB). Schon dieser Vergleich der Freiheitsstrafen des polnischen Rechts mit den deutschen Freiheitsstrafen zeigt, dass die in Art. $225 \$ 1$ poln. StGB neben der Todesstrafe und neben lebenslangen Freiheitsentzug angedrohte Freiheitsstrafe von mindestens fünf Jahren der Zuchthausstrafe des deutschen Rechts entspricht. Außerdem können deutsche Gerichte auch bei der Anwendung ausländischen Rechts nur auf eine nach dem deutschen Recht zulässige Strafe erkennen, und danach darf Gefängnis als Einzelstrafe gegen einen Erwachsenen höchstens bis zu fünf Jahren verhängt werden ( $\$ 16$ Abs. 1 StGB). Daher hat das Schwurgericht hier zutreffend nicht Gefängnis-, sondern Zuchthausstrafen für verwirkt erachtet. Deren Bemessung auf 15 Jahre für L. und auf 10 Jahre für R. ist aus Rechtsgründen nicht zu beanstanden.

c) Grundlage der Ehrenstrafe ist bei beiden Angeklagten nicht das deutsche Recht, wie das Schwurgericht angenommen hat, sondern Art. $47 \$ 2$ poln. StGB, dessen Voraussetzungen der Tatrichter nach dem Urteil ausdrücklich als gegeben angesehen hat. Dieser Fehler hat sich in dem Urteilssatz nicht ausgewirkt; der Senat braucht ihn daher nur hier in den Gründen richtigzustellen. 
Dem Angeklagten L. werden die bürgerlichen Ehrenrechte entsprechend der Beratung des Schwurgerichts auf zehn und nicht auf fünfzehn Jahre aberkannt, wie es in der Formel des angefochtenen Urteils irrtümlich heißt.

Danach sind die im Ergebnis erfolglosen Revisionen zu verwerfen. Das Urteil wird jedoch dahin berichtigt, dass die Verurteilung der Beschwerdeführer wegen gemeinschaftlicher vorsätzlicher Tötung auf Art. $225 \$ 1,47 \S 2$ poln. StGB gestützt wird und dass die bürgerlichen Ehrenrechte dem Angeklagten L. auf zehn Jahre aberkannt werden.

\section{Zur Rezeption des Urteils}

Auch wenn die zeitgeschichtlichen Umstände dieses Falles - anders als etwa bei den Urteilen in den Auschwitz-Prozessen - in der deutschen Strafrechtswissenschaft nicht weiter thematisiert worden sind, wurde (und wird bis heute) das Urteil doch als (Präzedenz-)Fall für die Verfassungsmäßigkeit der sog. Neubürger-Klausel wie auch für das Erfordernis einer restriktiven Interpretation genannt. Exemplarisch heißt es etwa seit Jahrzehnten in der knappen Erläuterung von Karl Lackner zu der streitgegenständlichen Norm des $\$ 7$ Abs. 2 StGB (bis zum 1.1.1975: $\$ 4$ Abs. 2 StGB a.F.): „Seine 2. Alternative (Neubürger) ist verfassungsrechtlich bedenklich, weil sie deutsches Strafrecht auf Täter für anwendbar erklärt, die ihm zur Tatzeit noch nicht unterworfen waren (aM BGHSt 20, 22); ein milderes Tatortrecht muss deshalb zur Begrenzung berücksichtigt werden “ ${ }^{10}$ Jüngst ist freilich wieder in der Literatur die Verfassungswidrigkeit der Neubürger-Klausel postuliert worden, ${ }^{11}$ da es mir hier vor allem um den rechtshistorischen Hintergrund dieses (Präzedenz-)Falles geht, möchte ich auf diese Diskussion hier aber nicht näher eingehen.

Eine andere zumindest bis zu den Ostverträgen offene Frage stellte sich angesichts des konkreten Tatorts im Raum Posen nicht, denn dieser gehörte - wie der BGH festhält - mindestens bis zum 31.10.1939 auch aus Sicht des damaligen Deutschen Reiches unzweifelhaft zu Polen und war nicht etwa bereits 1937 Teil des deutschen Reichsgebietes. Daher konnte vorliegend der Einfluss des damals noch im Verhältnis der Bundesrepublik Deutschland zu Polen - zwischen der DDR und Polen war der heutige Grenzverlauf bereits 1950 im Görlitzer Vertrag als polnische Westgrenze bestätigt worden - ungeklärten Status der von Polen nach Kriegsende übernommenen 1937 bereits zu Deutschen Reich gehörenden Gebiete auf das Strafanwendungsrecht außer Betracht bleiben.

10 So übernommen von Heger, in: K. Lackner, K. Kühl, StGB-Kommentar, 28. Aufl. 2018, \$ 7 Rn. 4.

11 Zehetgruber, ZIS 2020, 364, 377 ff.; dagegen etwa G. Werle, F. Jeßberger, Leipziger Kommentar zum StGB, 13. Aufl. 2019, § 7 Rn. 87. 
Mangels näherer Kenntnisse zu den konkreten Umständen der angeklagten Tat - wann fand diese genau statt und war das Territorium zu diesem Zeitpunkt bereits von Deutschland annektiert? - und den persönlichen Verhältnissen der Täter zur Tatzeit - waren sie zu diesem Zeitpunkt bereits Staatsangehörige des Deutschen Reichs oder noch als Teil der deutschen Minderheit Staatsbürger Polens? -, beschränkt sich der BGH wie schon das Schwurgericht Stuutgart in dubio pro reo auf die Annahme der für die Täter günstigsten Möglichkeit, so dass den rechtlichen Ausführungen die Option zugrunde liegt, dass der Tatort zur Tatzeit Ausland war und Täter wie Opfer damals Ausländer. Hätte sich an dieser Betrachtung bis zur Anklage nichts geändert, wäre deutsches Strafrechts freilich gar nicht anwendbar gewesen. Weil aber damals $₫ 4$ StGB und heute inhaltsgleich $\$ 7$ Abs. 2 Nr. 1 Alt. 2 StGB eine sog. Neubürger-Klausel enthalten, kann deutsches Strafrecht aufgrund des aktiven Personalitätsprinzips auch dann zur Anwendung kommen, wenn der Täter erst nach der Tat die deutsche Staatsangehörigkeit erworben hat oder auf andere Weise Deutscher im Sinne des Art. 116 Abs. 1 GG ist.

Neben den Deutschen kraft Staatsangehörigkeit gab und gibt es auch Deutsche kraft Volkszugehörigkeit, unabhängig davon, ob diese eine andere Staatsangehörigkeit haben. Soweit die Tat nach Inkrafttreten des Grundgesetzes am 23.5.1949 durch einen Volksdeutschen begangen worden ist, passt die Neubürger-Klausel auf ihn nicht, denn er war zur Tatzeit ja bereits Deutscher im Sinne von Art. 116 Abs. 1 GG. Platz für die Neubürger-Klausel ist für eine spätere Tatbegehung nur, wenn es sich nicht um Volksdeutsche handelt, die nach der Tat die deutsche Staatsangehörigkeit erworben haben. Da aber Mord in Deutschland seit 1979 nicht mehr verjähren kann, wäre es unverändert denkbar, dass jemand, der - ohne formal die deutscher Staatsangehörigkeit erworben zu haben - die Tat als Volksdeutscher in einem eroberten Gebiet begangen hat; dieser ist eben mit Art. 116 GG zu einem Deutschen im Sinne des deutschen Strafrechts geworden, so dass - weil dies ja nach der Tatbegehung erfolgt ist - deutsches Strafrechts aufgrund der Regelung des $₫ 7$ Abs. 2 Nr. 1 Alt. 2 StGB zur Anwendung berufen wäre.

Die Anerkennung auch von Volksdeutschen als Deutschen durch Art. 116 Abs. 1 GG und damit auch für das deutsche Strafrecht ohne deren dahingehender Willensbekundung und bei häufig anderer Staatsangehörigkeit hätte in einigen Fällen dazu führen können, dass auch polnische Staatsangehörige, die die Voraussetzungen von Art. 116 Abs. 1 GG erfüllt haben, bei einer Tatbegehung auf polnischem Staatsgebiet nach Inkrafttreten des Grundgesetzes als Deutsche zur Tatzeit i.S. von $₫ 7$ Abs. 2 Nr. 1 Alt. 1 StGB oder bei einer Tatbegehung zuvor aufgrund der Neubürger-Klausel auch deutschem Strafrecht unterliegen konnten. Denkbar - und nicht unwahrscheinlich - war, dass ein Angehöriger der deutschen Minderheit in Polen nur die polnische Staatsangehörigkeit besaß, aber in dem 1937 noch zum Deutschen Reich gehörenden Westteil Polens siedelte. 
Er wäre mithin neben seiner polnischen Staatsangehörigkeit auch Volksdeutscher und zugleich im vormaligen Reichsgebiet in dessen Grenzen von 1937 ansässig; als Volksdeutscher im Sinne von Art. 116 Abs. 1 GG wäre damit auch deutsches Strafrecht im Rahmen des Personalitätsprinzips auf ihn anwendbar gewesen. Hätte etwa ein Pole mit deutschen Wurzeln, wohnhaft in den vormaligen deutschen Ostgebieten, zwischen dem Kriegsende 1945 und dem Inkrafttreten des Grundgesetzes 1949 in Polen einen Polen getötet, wäre auf ihn als Volksdeutschen aufgrund von Art. 116 Abs. 1 GG nachträglich deutsches Strafrecht anwendbar. Und in den Jahren nach 1949 wären polnische Staatsangehörige deutscher Herkunft zugleich Volksdeutsche im Sinne von Art. 116 Abs. 1 GG, so dass auf alle ihre Handlungen im Ausland nach $₫ 7$ Abs. 2 Nr. 1 StGB (auch) deutsches Strafrecht anwendbar war, soweit nur die fragliche Handlung auch im Tatortstaat (etwa in Polen) strafbewehrt war.

Diese Konsequenz wurde in der bundesdeutschen Strafjustiz spätestens nach dem 1972 ratifizierten Warschauer Vertrag nicht (mehr) gezogen. Wiederum von Lackner kann man dazu lesen: „Jedoch werden Einwohner von Gebieten jenseits der Oder-Neiße-Grenze nicht mehr erfaßt, seit die Bundesrepublik im Warschauer Vertrag die Westgrenze Polens völkerrechtlich anerkannt hat. "12 Auf deutschstämmige Polen, die in vormals reichsdeutschen Gebieten lebten und außerhalb (West-)Deutschlands Straftaten begangen haben, war folglich bundesdeutsches Strafrecht zumindest ab 1972 nicht mehr aufgrund der Neubürger-Klausel anwendbar. Hatten sie Polen - wie nicht wenige der zunächst noch dort verbliebenen Angehörigen der deutschen Minderheit - bis in die 1980er Jahre verlassen und in der Bundesrepublik Deutschland als Volksdeutsche sofort die deutsche Staatsangehörigkeit erhalten, wäre allein deshalb vor 2000 eine Auslieferung nach Polen nicht zulässig gewesen, so dass - sollten sie vor ihrer Ausreise in die Bundesrepublik Deutschland in Polen straffällig geworden sein zumindest nach dem völkerrechtlich anerkannten und in $₫ 7$ Abs. 2 Nr. 2 StGB niedergelegten Grundsatz der stellvertretenden Strafrechtspflege auch hierzulande ein Strafverfahren möglich war; dass daneben - je nach Tatzeitpunkt - der Täter auch vor Erwerb der deutschen Staatsangehörigkeit aufgrund von Art. 116 Abs. 1 GG Deutscher entweder bereits war oder nach der Tatbegehung (wenn diese vor dem 23.5.1949) es geworden ist, hätte zwar nach $\$ 7$ Abs. 1 Nr. 1 StGB ebenfalls die Anwendbarkeit deutschen Strafrechts begründen können, bewirkte damit aber jedenfalls keine Ausdehnung des bundesdeutschen Strafanwendungsrechts in einen völkerrechtlich problematischen Bereich. Wer dagegen als deutschstämmiger polnischer Staatsangehöriger ab 1972 in die Bundesrepublik Deutschland einreiste, ohne deren Staatsangehörigkeit zu erwerben, konnte nicht (mehr) allein wegen seiner deutschen Volkszugehörigkeit als Deutscher im Sinne des Starfanwendungsrechts behandelt werden.

12 K. Lackner, StGB mit Erläuterungen, 19. Aufl. 1991, Vor $\$ \$ 3-9$ Rn. 7. 
Mit Blick auf die DDR gab es in der bundesdeutschen Strafrechtsprechung auch immer wieder das Problem, inwieweit auf DDR-Bürger, die ja zumindest im Sinne von Art. 116 Abs. 1 GG auch Deutsche waren, für Straftaten auf dem Gebiet der DDR (bundes-)deutsches Strafrecht anwendbar sein sollte. Soweit innerhalb eines Hoheitsgebietes - wie z.B. innerhalb der Bundesrepublik Deutschland - an verschiedenen Orten unterschiedliches Strafrecht gilt, geht es nicht um das in $\$ \$ 3 \mathrm{ff}$. StGB geregelte internationale Strafrecht bzw. Strafanwendungsrecht, sondern um ein sogenanntes interlokales Strafrecht. Ob ein nur in einem Teilgebiet eines Landes geltendes Strafrecht auf einen Sachverhalt anwendbar ist oder nicht, richtet sich primär nach dem Territorialitätsprinzip, ${ }^{13}$ so dass etwa nur in Bayern (und nicht auch in Berlin) strafbare Handlungen in Deutschland nur, aber auch immer dann verfolgt werden können, wenn sie innerhalb Bayerns begangen worden sind. Eine Konsequenz daraus ist, dass das nur in einem Teilgebiet eines Landes geltende Strafrecht auch durch die Strafgerichte in anderen Landesteilen angewendet werden kann, so dass die beispielhaft gedachte bayerische Strafnorm auch dann durch die Berliner Strafjustiz verfolgt werden könnte (und angesichts des Legalitätsprinzips grundsätzlich auch müsste), wenn das gleiche Verhalten - wäre es in Berlin vorgekommen - angesichts im dort geltenden Recht nicht strafbewehrt wäre. Dieses interlokale Strafrecht hatte etwa während der NS-Zeit große Bedeutung in Ansehung von Straftaten in den ab 1939 annektierten Teilen Polens, für die teilweise eigene Strafnormen erlassen worden sind. ${ }^{14}$

Diese Idee ist zunächst in der westdeutschen Rechtsprechung für das Verhältnis des bundesdeutschen Strafrechts zu in der DDR spielenden Sachverhalten erwogen worden; die Rede war insoweit von einem „interzonalen Strafrecht“ . ${ }^{15}$ Allerdings setzt das interlokale Strafrecht voraus, dass innerhalb des gesamten Gebiets, in dem nur an einzelnen Orten geltende Strafnormen gerichtlich durchgesetzt werden können, „eine grundsätzliche vom gegenseitigen Vertrauen beherrschte Anerkennung der verschiedenen Teilrechtsgebiete für den gesamten Bereich" (bei aller Unterschiedlichkeit) voraus, ${ }^{16}$ was angesichts der fundamentalen rechtlichen Unterschiede beider deutscher Staaten im kalten Krieg sicher nicht gegeben war. Wirklich an Bedeutung gewonnen hat das interlokale Strafrecht daher erst nach der deutschen Wiedervereinigung, als aufgrund des Einigungsvertrages ab 1990 im Beitrittsgebiet teilweise noch aus der DDR stammende Strafnormen in Geltung geblieben sind. ${ }^{17}$

13 G. Werle, F. Jeßberger (Fn. 11), Vor $\$ 3$ Rn. $420 \mathrm{ff}$.

14 H.-H. Jescheck, T. Weigend, Strafrecht Allgemeiner Teil, 5. Aufl. 1996, \$ 20 I; G. Werle, F. Jeßberger (Fn. 11), Vor $\$ 3$ Rn. 417.

15 Vgl. H. Tröndle, Leipziger Kommentar zum StGB, 10. Aufl. 1985, Vor Rn. 89.

16 So H. Tröndle (Fn. 15), Vor $\$ 3$ Rn. 86.

17 Dazu näher G. Gribbohm, Leipziger Kommentar zum StGB, 11. Aufl. 1992, Vor $₫ 3$ Rn. 403 ff. 
Ähnlich wie mit Blick auf die vormals deutschen Teile Polens hat sich insoweit aber die Situation durch die Ostverträge geändert, denn spätestens mit dem Inkrafttreten des Grundlagenvertrags von Bundesrepublik Deutschland und DDR im Jahr 1973 wurde die DDR seitens der Bundesrepublik Deutschland im Sinne des Völkerrechts als eigener Staat anerkannt. Spätestens ab diesem Zeitpunkt und bis zur Wiedervereinigung wurden die Regelungen der $\$ \$ 3 \mathrm{ff}$. StGB und damit des internationalen Strafrechts jedenfalls der Sache nach auch im Verhältnis zur DDR angewandt. ${ }^{18}$

\section{Zum Schluss}

Die Neubürger-Klausel ist bis heute Teil des geltenden deutschen Strafanwendungsrechts. Praktisch hat sie aber so gut wie keine Bedeutung mehr. Gleichwohl entzündet sich an der Frage einer Erstreckung des deutschen Strafrechts auch auf Deutsche, die dies erst nach der Tatbegehung geworden sind, immer noch manche Grundsatzdiskussion. Würde man diese Klausel ersatzlos streichen, bliebe angesichts der deutschen Staatsangehörigkeit zumindest außerhalb der Europäischen Union auch nach der Einschränkung von Art. 16 GG zumindest ein Auslieferungshindernis, so dass deutsches Strafrecht jedenfalls deswegen anwendbar bleibt.

In der Diskussion um die Neubürger-Klausel in $\$ 7$ Abs. 1 Nr. 1 StGB wird bis heute regelmäßig auf BGHSt 20, 22 verwiesen. Diesem zugrunde lag allerdings eine Kombination dieser Klausel mit der Zusprechung der DeutschenEigenschaft ab 1949 durch Art. 116 Abs. 1 GG. Eine solche Konstellation wird zwar mit der Zeit immer unwahrscheinlicher; angesichts des in den letzten zehn Jahren wieder aufgeflammten Verfolgungseifers etwa gegen Wachleute in den Vernichtungslagern erscheint es aber nicht ausgeschlossen, dass angesichts der Tatbegehung vor 1949 die Neubürger-Klausel auf Grundlage von Art. 116 Abs. 1 GG bis heute einer Strafverfolgung durch die deutsche Strafjustiz zugrunde liegen könnte.

\section{Bibliography}

Dalcke A., Fuhrmann E., Strafrecht und Strafverfahren, Berlin 1961.

Geilke G., Die polnische Strafgesetzgebung seit 1944, Berlin 1955.

Geilke G., Staatsangehörigkeitsrecht von Polen, Berlin 1952.

Hegler A., Prinzipien des Internationalen Strafrechts, Berlin 1906, Nachdruck 2012.

Jeßberger F., Der transnationale Geltungsbereich des deutschen Strafrechts, Tübingen 2011.

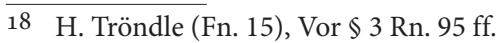


Jescheck H.-H., Zur Reform der Vorschriften des StGB über das Internationale Strafrecht, „Internationales Recht und Diplomatie“ 1956.

Jescheck H.-H., Weigend T., Strafrecht Allgemeiner Teil, 5. Auflage, Berlin 1996.

Lackner K., StGB mit Erläuterungen, 19. Auflage, München 1991.

Lackner K., Kühl K., Strafgesetzbuch, 29. Auflage, München 2018.

Leipziger Kommentar Reichs=Strafgesetzbuch, Band 1, 5. Auflage, Berlin und Leipzig 1933.

Leipziger Kommentar Strafgesetzbuch, Band 1, 10. Auflage, Berlin 1985.

Leipziger Kommentar Strafgesetzbuch, Band 1, 11. Auflage, Berlin 1992.

Leipziger Kommentar Strafgesetzbuch, Band 1, 13. Auflage, Berlin 2019.

Meyer H., Lehrbuch des Deutschen Strafrechts, 5.Auflage, Erlangen und Leipzig 1895.

Müller S., John T., Die Mörder sind unter uns: Der Ulmer Einsatzgruppenprozess 1958, Stuttgart 2008.

Riedel J., 50 Jahre Zentrale Stelle in Ludwigsburg. Ein Erfahrungsbericht über die letzten zweieinhalb Jahrzehnte, Vierteljahreshefte für Zeitgeschichte, 2008.

Rieß P., Entwicklungstendenzen in der deutschen Strafprozessgesetzgebung seit 1950, ZIS 2009.

Sinn A., Jurisdiktionskonflikte bei grenzüberschreitender Kriminalität, Osnabrück 2012.

von Bar L., Gesetz und Schuld im Strafrecht: Fragen des deutschen Strafrechts und seiner Reform, Berlin 1906.

von Olshausen J.,Niethammer E., Kommentar zum StGB für das Deutsche Reich, Berlin 1927.

Werle G., Wandres T., Auschwitz vor Gericht, München 1995.

Zehetgruber C., Eine kritische Betrachtung der Anknüpfungspunkte des § 7 StGB, ZIS 2020.

\section{SUMMARY}

The judgment of the Bundesgerichtshof (Federal Court of Justice) from the $4^{\text {th }}$ September 1964 and the "New Citizenship Clause" as part of the German law of jurisdiction in criminal cases - a German-Polish case and its implications for the legal development till today

The "New Citizenship Clause" is still part of current German criminal law. In practice, however, it has virtually no significance anymore. Nonetheless, the question of extending German criminal law to Germans who have become subject to it only after the commission of a crime is still the subject of debate. If this clause were to be deleted without substitution, in view of German citizenship, at least outside the European Union, at least one obstacle to extradition would remain even after the restriction of Article 16 of the Basic Law, so that German criminal law would remain applicable for this reason at any rate. In the discussion around the new citizen clause in section $7 \mathbb{\$} 1$ No. 1 of the German Criminal Code is referred to judgment of the 4th 
September 1964 the German Federal Court of Justice until today regularly. However, this was based on a combination of this clause with the attribution of German citizenship from 1949 onwards by Article $116 \$ 1$ of the Basic Law. Although such a constellation is becoming increasingly unlikely over time, in view of the eagerness to prosecute that has flared up again in the last ten years; for example, against guards in the extermination camps, it does not seem impossible that, in view of the commission of the crime before 1949, the Neubürger clause based on Article 116 $\mathbb{} 1$ of the Basic Law could still form the basis of criminal prosecution by the German criminal justice system today. 